\title{
A Review of Blockchain based Educational Projects
}

\author{
Bushra Hameed $^{1}$, Muhammad Murad Khan*2, Abdul Noman ${ }^{3}$, M. Javed Ahmad ${ }^{4}$, \\ Prof. Dr. M. Ramzan Talib ${ }^{5}$, Faiza Ashfaq ${ }^{6}$, Hafiz Usman ${ }^{7}$, M. Yousaf ${ }^{8}$ \\ Department of Computer Science, Govt College University Faisalabad, Faisalabad, Pakistan
}

\begin{abstract}
Blockchain is a decentralized and shared distributed ledger that records the transaction history done by totally different nodes within the whole network. The technology is practically used in the field of education for record-keeping, digital certification, etc. There have already been several papers published on this, but no one can't find a single paper covering the blockchain-based educational projects. So, There is a gap of latest trends to education. Blockchain-based educational projects resolve the issues of today's educators. On that basis, we conclude that there is a need for conducting a systematic literature review. This study, therefore, reviews the artistic gap between these two based on educational projects. For this purpose, the paper focuses on exploring some block-chain based projects and protocols that are used in these projects. It also analyses the block-chain features that are being used and the services are offered by the existing educational projects using block-chain features to improve the execution of this technology in education.
\end{abstract}

Keywords-Blockchain; educational-project; education; digitalcertification; record-keeping

\section{INTRODUCTION}

Block-chain technology was developed first for Bitcoin crypto-currency. The attraction in block-chain has been catching the attention since the idea was coined in 2008 [1], [2]. It is a decentralized and self-regulating technology [3], keeps an open record of all transactions or computerized events that have been executed and shared among partaking parties [4]. Every transaction on a block-chain is verified with digital signatures that demonstrate its originality. Since the use of encryption and digital signatures, the information stored on the block-chain is sealed and can not be changed [5], [6]. Through this, data can be handled and sorted out in another manner: open-source, verified, and shared without the need for a central charge [7].

Because of its unchanging nature, transparency, and security of all transactions implemented in a block-chain, this innovative technology is used in many applications such as health care, education, governance and the business industry [8], [9], [10], [11].

In today's era, block-chain highlights the major and burning issues regarding education and provides suitable solutions for them. The certificate is considered to be depicting the pictorial images of the ability of a person, which was a conventional method of displaying grades on paper. Now, this technology has provided a new trend of imprinting the digital certificate [12]. Unlikely paper certificates, the digital certificates has simplified the process of management [13], [14], [15], [16], [17], [18]. Block-chain technology also causes to reducing degree fraud and improve the system for calculating scholarships for students,evaluation system and salaries for teachers and will provide a fair mechanism with complete transparency for funding projects of education and its grants [15], [19].
For the conversion of today's education system, different educational projects introduced for the user's benefits, but we have to see that these educational projects are not discussed majorly in the single study. There is a gap for describing the latest educational trends. This study views the gap between block-chain in education to the latest educational trends as well as their implementations(applications). To fill this gap, this paper describes some vital blockchain-based educational projects and protocols.

It also analyses the blockchain features that are being used and the services are offered by the existing educational projects using blockchain features to enhance the implementation of block-chain technology in education. This review paper includes some important block-chain educational models from 2013 to 2018. These are Edgecoin, Sucesslife, TeachMePlease, SonyGlobalEducation project, Blockcert, Gradbase, Origin Stamp, Echolink and Tutellus. But there are still some problems, like, scalability issues [20], [21], [11], [3], [9]. Scalability is defined as: "The times is taken to place a transaction within the block, The time is taken to reach a consensus, The time taken to put a transaction in the block".

The paper is divided into 5 sections. Section 2 is concerned with the literature review and describes the features of blockchain. Section 3 explores the Research methodology, Research questions and Search strategy is mentioned. Section 4 covers the results of the research question and the last section concludes the restriction of our review.

\section{REVIEW OF LITERATURE}

This segment of this paper describes a few ideas and theories related to existing studies in the field of blockchain based educational projects and highlights some primary features of block-chain. It also constructs a correlation between the existing secondary studies

\section{A. Blockchain}

A blockchain in the simplest term is an immutable record of data that cannot be changed once it becomes part of a blockchain hosted on a decentralized network [11], [22]. Since all record is handled by a group of nodes not owned by a single entity, it is nearly impossible to tamper and update the record on all objects of the network. Every one of these Blocks is bound to use cryptographic proof for signing and verifying the transactions. Nodes start the procedure by making the blocks. Block is hash-checked by each node on the network for verification. The verified block is added to the chain which is archived by the whole system, making not just a tamper-proof record but rather a particular record with a unique history [4], [23], [24], [25]. 


\section{B. Types of Blockchain}

The basic types of blockchain are as follows:

Permissioned Blockchain: Permissioned blockchains act as closed ecosystems, where users are not freely capable of joining the community, see the records, or issue transactions of their own. Permissioned blockchains are favored by using centralized organizations, which leverage the strength of the network for his or her own, inner business operations. Employer consortiums also are likely to hire personal blockchains to file transactions securely, and exchange facts among one another.

Permissionless Blockchain: A permissionless blockchain is open to everyone. It allows every user to create a non-public deal with and start interacting with the network, by way of submitting transactions, and subsequently adding entries to the ledger.

Hybrid Blockchain: The blockchain that utilizes the combination of both private and public blockchain. The hybrid network means permissionless blockchain is used where a permissioned blockchain is hosted. This blockchain is not open to everyone but quietly provides basic features like integrity, security, and traceability.

\section{Blockchain Features}

The Basic features of blockchain are as follows:

Decentralized: Blockchain is a common open record wherein all nodes are associated with one another in a network [9], [10], [26], [27], [1]. Decentralization refers to the procedure of data confirmation, storages, preservation and transmission on the blockchain which depend on distributed framework structure [28], [30].

Traceability: All transactions on the blockchain are indexed in sequential order, and a block is associated with two adjoining blocks by a cryptographic hash. A total chain of a block is kept up by mining pools, which gives cloud-based sites to investigating the block [9], [28], [30].

Consensus mechanism: A fault tolerant mechanism is a consensus mechanism that is used in PC and blockchain frameworks to accomplish the agreement on a single data of network state among distributed processes or multi-agent frameworks. It is helpful in record keeping. POW, POS, and DPOS are the few procedures of consensus mechanism [9], [32].

Currency: In this revolutionary era, bitcoin was the first digital currency of all cryptocurrencies that have been proposed. Cryptocurrency whose property is blockchain technology which is a digital or virtual currency that ensures end-to-end transactions making it protected and trustworthy. Different mining algorithms are used for the formation of these currencies. Thus, the combined product of blockchain and cryptocurrency can be used in several aspects such as Accounting and Finance [33], [9], [28].

Smart contract: A smart contract is a necessary blockchain protocol that allows developers to code the financial agreement on the blockchain, which is activated by the involved parties [30], [9], [34], [28], [35]. It did not just decrease outsider cost" in conventional transactions yet also significantly ensures the transaction security and quality.

Immutability: When data is entered, it can't be changed [36]. Successful tampering (make unauthorized alterations) would need to change more than 51 per cent of the records put away in the system [9], [28], [29].

Fig. 1 represents the name of basic features of blockchain.

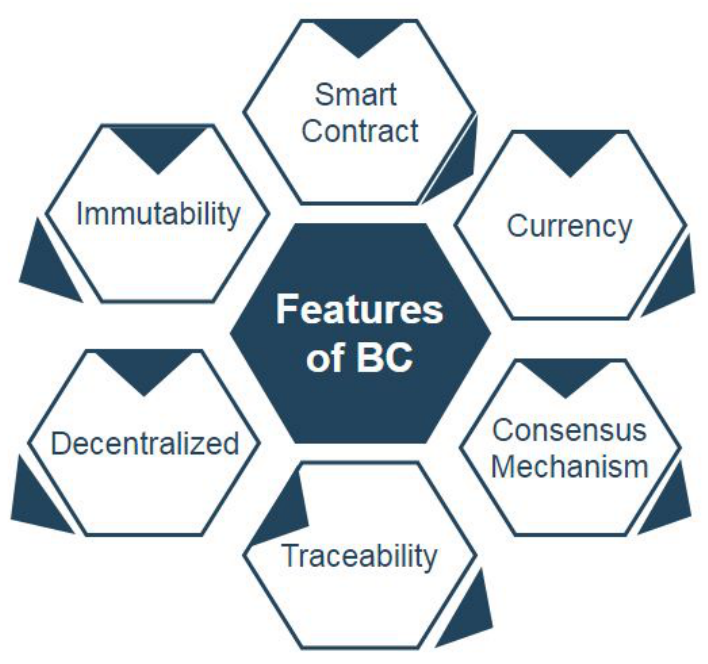

Fig. 1. Basic Features of Blockchain

\section{Blockchain Application in Education}

Blockchain is a technology that has applications within the world of learning at the individual, institutional, group, national, and international levels. It is applicable in all kinds of frame of reference: Schools, colleges, universities, cooperates, studentship and knowledge bases, instead of old hierarchical structures, the technology becomes the main target, with trust migrating towards the technology, not the institutions. It extremely is decentralized technology (Donald Clark). Some of the Blockchain-based educational projects used across the world are as follows:

Edgecoin: Edgecoin is inside the strategy to structure a Dapp stage for cost bringing down, efficient and Fraud protected ensured great arrangements. It stores academic documents like degrees and accelerated the tip of the paper-based system. This cut expenses for students since they don't need to pay for a legal official. Edgecoin, the innovative reforms the education trade totally, conveyance interruption of the fouled and non-current education, its certifications and validated the procedure. So they're defensive against Fraud, damages, and losses of any kind [36].

Sony Global Education (SGE): Sony Global Education is a new educational platform that came into existence by the partnership of IBM in which we secure and share the record of a student by using blockchain. On 10th August 2017, Sony Corporation and Sony global education announced that we would form such a system on which blockchain technology 
will be applied specifically in the Education system. In 2018, Sony will start to establish its service offerings that will start with the global math challenge whose purpose will be to bring 150000 participants from all over the world cooperatively [28], [16], [17], [37], [38].

Tutellus: Tutellus is a new blockchain platform that is expecting to solve the current educational costs for collegelevel students and in fact paying students for learning. In 2016, online education was having a specified value of USD 165 billion. It is insufficient to complete the need for education in the whole world. Whether the number of graduates who remain unemployed is over 300 million, there is an increasing number of educated people in the market and opposition to the fact, the number of vacancies or posts is gradually decreasing to a great extent. In Europe, 80 percent imbalance between job supply and demand is expected, specifically affecting the technology sector. Resultantly, there is increasing awareness that educational institutions are lacking up to date knowledge according to the employer's needs [?]. On the other side, developing countries have millions of unemployed people who are getting access to the Internet with the help of their mobile phones. They can join this platform because, for the poorest, it's rarely affordable. A new method also required to tackle the key problems that market faces that current educational institution has tried to resolve [39], [40], [41].

TeachMePlease (TMP): Teachmeplease (TMP) provides a database of learning institutions for both online and offline schools, as well as different educational programs, from group learning classes for kids to individual sessions with the personal instructor. It needs to work on the delicate data, for example, courses, assignments, solutions, and grades. TMP used permission less blockchain, as Ethereum or EOS, would require revealing this data to the general public, though the agreeable ones, like Hyperledger, need open unquestionable status. TMP promises "a quality platform that provides easy to use service for each of our clients" [42], [9].

SuccessLife: From success resources, Successlife is that the original front runner product, the world-leading seminar and workshop organizer. To make personal and professional development available to as many people as possible. Success Resources founded the blockchain company SuccessLife. Around 543000 new businesses begin every month, of which the SuccessLife primary target. More than 50 percent of the working population work in a private venture small business. Fifty-two percent of all small businesses are home-based.

Blockcerts: Blockcerts is an open-source blockchain platform that was developed for the educational record by MIT Lab with the collaboration of the learning machine. The wallet app is used to encrypt the student information. The main purpose is to produce secure, validate and issue e-certificate by using bitcoin blockchain technology [43], [14].

GradBase: Gradbase is an educational record verification system based upon the bitcoin blockchain technology. It provides an online profile system simply and inexpensively. This system provides a QR code to be included in student CVs. For the enhancement of the user experience, it provides a web browser extension with LinkedIn support. Universes can easily $\log$ in the web app for the updation, cancellation the qualifications in the appropriate procedure.
Echolink: Echolink is the abbreviation of "Education Carrier Skills Human Capital Opportunity Link". Echolink is a blockchain-based system that organizes a relationship between educational entities, i.e., Students, teachers, and other organizations. It stores the verified data of individual related to the professional career in the hashed and unalterable format on the permission-less blockchain. All the information are provided by trusted sources. The identified projects are further compared, and related synthesis is presented in the following sections [43], [44].

Origin-Stamp: Origin-Stamp is secure time-Stamping for ensuring the security of digital data. It determines the eccentric fingerprints of the client data and keeps the fingerprints permanently in the Bitcoin blockchain technology [38].

The identified projects are further compared, and related synthesis is presented in the following sections:

\section{E. Compared Secondary Studies}

Blockchain technology is, no doubt has got a tremendous appreciation all over the world by its magic and rapid progress. However, while searching for secondary studies, we found just a couple that talks about the latest educational projects. Firstly,

TABLE I. COMPARED SECONDARY STUdiES

\begin{tabular}{lccc}
\hline No & $\begin{array}{c}\text { Blockchain } \\
\text { Features }\end{array}$ & $\begin{array}{c}\text { BC Based } \\
\text { Educational Projects }\end{array}$ & $\begin{array}{c}\text { Projects } \\
\text { Services }\end{array}$ \\
\hline Grech [8] & 3 & 4 & Gap \\
\hline Yumna [43] & 6 & 3 & Gap \\
\hline This Study & $6+3$ & 10 & Discussed \\
\hline
\end{tabular}

a report that was published by the European Commission was found, and we have recognized that in this study there is only a few latest project described in short length, rest latest are not described and even not touched. Second, a systematic literature review that was published in Springer, which discussed the application of blockchain in education, Table I enlists secondary studies concerning the educational project.

\section{RESEARCH Methodology}

A Systematic Literature Review is defined as "A method of gathering, identifying and interpreting all available research in order to answer a specific research question" [46], [9]. In this study, we follow the guidelines provided by "Barbara Kitchenham" [45]. The results of this examination help us to distinguish research area related to Blockchain technology and conceivable research gaps. According to Kitchenham, SLR comprises on three main stages as given below:

- Planing the review.

- Conducting the review.

- $\quad$ Reporting the review.

In this research, we follow above these steps for completing research; Fig. 2 shows the steps that are further divided into more stages. 


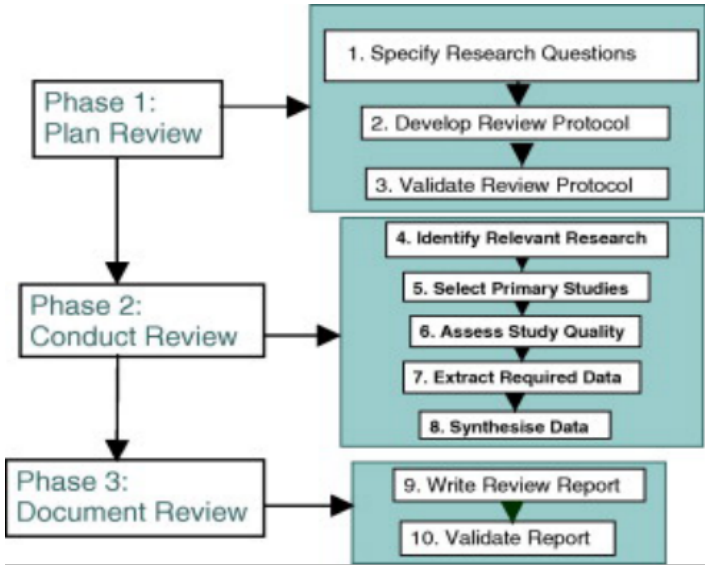

Fig. 2. SLR Methodology

\section{A. Need of Conducting SLR}

The properties of secondary studies as per the classification of these studies are illustrated in Table I. After analyzing the current studies, it is recognized that few holes have not been talked about yet and should to be filled for further suggestions. During the process of conducting this study, there are only a few studies discovered that have addressed the blockchainbased educational projects. But it was not done unanimously in single time paper. Henceforth, this led us to conduct this study to precisely accomplish our goals, and the quantitative research approach are used.

\section{B. Research Question}

The first part of this systematic literature review is to state the research questions. However, the following section is concentrated on the research questions:

RQ1: What are some of the blockchain projects related to education? (Aim: To illustrate some blockchain project that was developed for educational Motive)

RQ2: Which Protocols are used by these projects? (Aim:To)

RQ3: Which blockchain features are used by these projects? (Aim: To extend the blockchain features which are not discussed in existing studies \& elaborate on the implementation of blockchain features used in existing projects)

RQ4: What services are being offered by the existing educational projects using Blockchain features? (Aim: To highlight the similar and dissimilar services to enhance the implementation of blockchain technology in education.)

\section{Search Strategy}

Blockchain plays an essential role in the field of education. We studied different analysis papers from Google scholar. On Google Scholar, we tend to search the keywords like 'Blockchain', 'education' and 'review'. As a result, 200 papers were found on those subjects. After studying, we chose those papers which were related to our keywords. We inspected various research papers, reports an article published in different journals and conferences.

\section{Inclusion and Exclusion Criteria}

For inclusion, 35 papers were collected that were related to the keyword and Research question. After reading the abstract and conclusion, we selected 11 primary studies for data extraction, which were about Blockchain based educational projects as per the need of the latest educational trends. Other papers were eliminated because of the content of those papers about the "Blockchain in education" whereas they were not related to the topic "Blockchain-based educational project". Table II presents the papers which were selected.

TABLE II. PAPER With REFERENCE ID

\begin{tabular}{|c|c|c|}
\hline$I D$ & Publish year & Paper name \\
\hline [14] & 2018 & Ethereum Use Cases \\
\hline [55] & 2018 & $\begin{array}{l}\text { Blockchain Based Professional Networking } \\
\text { and Recruiting Platform }\end{array}$ \\
\hline [17] & 2018 & $\begin{array}{l}\text { CredenceLedger: A Permissioned blockchain } \\
\text { for Verifiable Academic Credentials }\end{array}$ \\
\hline$[54]$ & 2017 & $\begin{array}{l}\text { Stampery Blockchain Timestamping } \\
\text { Architecture (BTA)-Version } 6\end{array}$ \\
\hline$[51]$ & 2018 & $\begin{array}{l}\text { OriginStamp: A blockchain-backed system } \\
\text { for decentralized trusted timestamping }\end{array}$ \\
\hline$[18]$ & 2018 & Disciplina: block-chain for Education \\
\hline$[30]$ & 2016 & $\begin{array}{l}\text { Participation and sharing economy: The } \\
\text { Spanish case of compartirmola }\end{array}$ \\
\hline$[52]$ & 2018 & $\begin{array}{l}\text { The Changing Tide of Corporate Social } \\
\text { Responsibility }\end{array}$ \\
\hline [9] & 2018 & $\begin{array}{l}\text { Exploring block-chain technology and its } \\
\text { potential applications for education }\end{array}$ \\
\hline$[10]$ & 2018 & $\begin{array}{l}\text { Blockchain for education: lifelong learning } \\
\text { passport }\end{array}$ \\
\hline [49] & 2018 & $\begin{array}{l}\text { Historico-Sociological Analysis of the } \\
\text { Sharing Economy from its Early Digital } \\
\text { Foundations to the Present }\end{array}$ \\
\hline
\end{tabular}

\section{E. Data Extraction}

After being shortlisted, data extracting process was applied on studies. In this stage, the appropriate data was selected from the shortlisted primary studies concentrating on blockchain based educational projects and basic features followed by identified projects. The extracted data was then used for analysis purpose. 12 primary studies covered the educational projects [36], [44], [43], [55], [38], [42], [41], [39], [28], [14], [40], [17].

\section{F. Publication Trend}

Since the research on Blockchain was started in 2008, From that time, the adoption and advancement of this technology increased at a greater rate. However, the very first paper that was discussed in the Blockchain-based educational project was found to be published in 2017. But no paper was found explicitly focusing on the identified educational projects.

\section{RESUlTS}

The result section is divided into three parts. The first part classifies some of the blockchain projects related to education. The second part describes the features of Blockchain that are used by these projects. The third subsection highlights the common and uncommon services of identified educational projects, and the fourth section describes the future direction. 


\section{A. RQ1: What are Some of the Blockchain Projects related to Education?}

Blockchain technology has brought a revolution in education. Our first research question is related to projects that are selected by studying different review papers. This review includes some important educational projects from 2017 to 2018, as shown in Fig. 3. These are Edge-coin, success life, Teach Me Please, Sorry Global Education Project, Echolink, Gradbase, OriginStamp, and Tutellus. With the general intro-

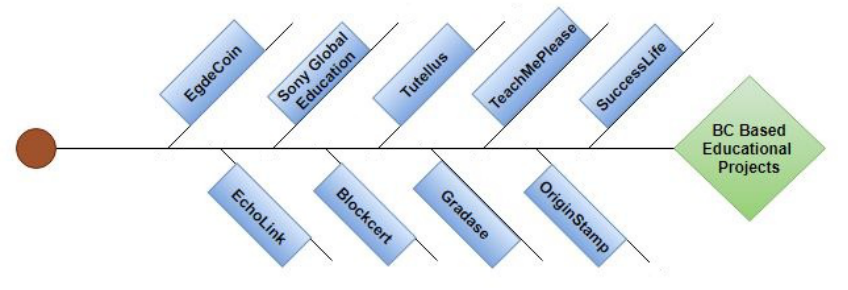

Fig. 3. BC Based Educational Projects

duction, it appeals to find out the reason behind this evolution. Our contributions consist of:

- Why we need blockchain-based educational projects?

Needs of blockchain-based educational projects: Today universities, individuals and employers face multiple challenges that include degree fraud, education verification, and problem translating and comparing the same degrees across different learning institutions [47]. Additionally, printing, authenticating, and storing degree and certification are the high costs for universities and schools in many parts of the world. While e-learning becomes the more and more popular, popular, verification of certification and validation of skills remains as there is no standard guideline that is accepted by all companies, countries, and institutions. The inability to validate skills continues to contribute to the large gap in the workforce. The multi-billion-dollar education industry needs solutions that make it easier to validate, verify, aggregate an individual's "learning record", making it easier to apply for the job and hire a qualified candidate.

We need a solution to all the challenges. So, blockchainbased educational projects resolved all the issues because blockchain is a safe, fast, the cost-efficient way to structure the records and data such as colleges degree, e-learning certificate, and much more. Blockchain enables products and services will disrupt the education system.

\section{B. RQ2:Which Protocols are being used by these Projects?}

All the educational projects have been built by following the blockchain technology whose main aim is to promote the real sense of education and check out all the hurdles and issues which comes in its way as threads; It provides a rational way out and solutions for all these issues. The major problems which emerge in the way of education are these:
Lack of educational data protection/security, too much traditional paperwork the long and inefficient certification process, insufficient ways of storing data, unsecured and non-immutable record keeping, etc. [27]. In the third world countries, the most common and root cause of illiteracy is poverty. The most significant achievement of the models, they not only provide the skills to the learners and also gives them a chance to improve their economical positions [22]. Table III represents the educational projects and technologies that are used by these projects.

TABLE III. DiffEREnt Educatioal Projects AND Used TECHNOLOGY

\begin{tabular}{lll}
\hline S:NO. & Models & Technology \\
\hline 1 & Edge-Coin & Proof of Scale (POS) \\
\hline 2 & TeachMePlease & DPoS \\
\hline 3 & SuccessLife & BOLT \\
\hline 4 & SonyGlobalEducation & HyperLedger Fabric \\
\hline 5 & Tutellus & Sharding \\
\hline 6 & Echolink & PoS \\
\hline 7 & OriginStamp & POW \\
\hline 8 & Gradbase & POW \\
\hline 9 & Blockcerts & POW \\
\hline
\end{tabular}

Edge-Coin stores educational documents like degrees and course certificates on its block for third parties to directly confirm their validity. This cut costs for students since they don't have to pay for a notary. It also reduces costs for institutions as it eliminates bureaucracy and paperwork discuss in Section II.

POS: Edge-Coin and EchoLink have used a proof of stake (PoS) as opposed to the standard Proof of Work (PoW). PoS is just a select few nodes, for the most part yet not chosen by the network on the quantity and age of their stack. By choosing a select few individuals to oversee the site, the transmission of smart contracts goes faster, Edge-Coin \& EchoLink can deal with its platform all the more speedily [48], [52], [49], [51].

POW: Blockcerts, Gradbase, OriginStamp, and Stempery have used a Proof of Work (POW). This Protocol used to validate the transactions. Through this Protocol, miners compete against each other to complete the transaction and get rewarded after solving cryptographic puzzles that have required more processing power.

DPoS: TeachMePlease used a DPoS Technology. Delegated Proof of Stake (DPoS) is a consensus mechanism algorithm maintaining undeniable agreement on reality across the network, authorizing transactions and providing a digitally democratic platform. Lisk has chosen it as its Protocol of choice due to its marvelous reputation and performance. Delegated ProofProof of stake uses voting in real-time combined with a scheme of name to achieve consensus. It is seen to be the smallest amount of centralized consensus protocol compared to all because it is most comprehensive of all. Each token holder will exercise a degree of influence regarding what happens on the network.

The TMP blockchain architecture divides itself into two layers: private and public. Private layer: This layer is used to store private and personal user data as well as those materials that are protected by copyright [42]. Public layer: This layer is used to provide access to the data that verifies the integrity of 
TABLE IV. FEATURES ARE USED BY THESE PROJECTS

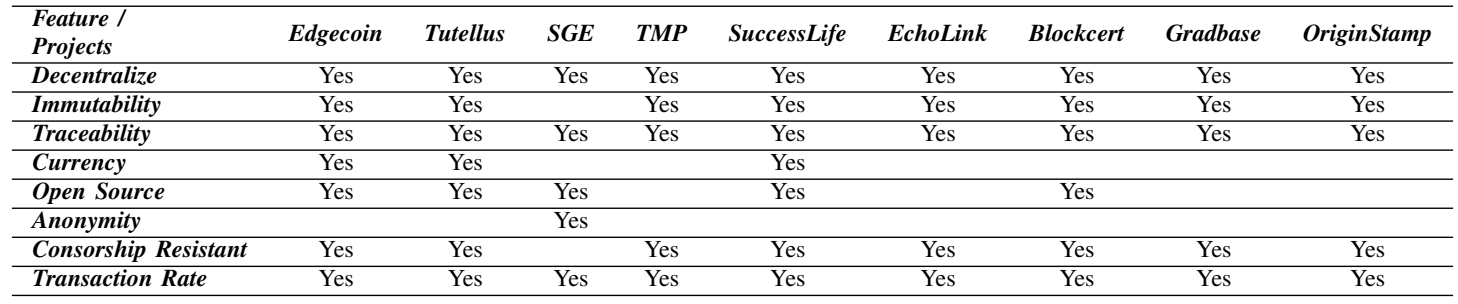

private data and the reliability of the data store by the network [42].

BOLT: To make professional and personal development available to as many people as possible, success Resources founded the blockchain company SuccessLife. SuccessLife used a BOLT Technology. The BOLT technology creates an entry in the Blockchain and enables Provenance.

- The interest in level, as well as search history of the user, has been captured in the level blockchain. This process will give better know-how and understanding of student's interests.

- The viewing of a demo step will create an entry in the blockchain lesson level that would certainly help the trainer identifying how many times his lessons were requested for a demo.

Hyperledger: The Sony Global Helpledger is a generalpurpose business platform that Supports various educational institutions for educational record storage and digital certificates. The solutions give an Application Programming Interface (API) to customize the attributes of data collections of student's records and control use rights for educational institutions [50], [35], [10], [53], [37].

Sony hyperledger solves the scalability problem by separating the standard PoW transaction processing that is performed at one go into three phases: building the blocks, ordering the blocks, and validating each of these blocks.

Sharding: Sharding is a type of database partitioning that separates more massive databases into smaller, faster, more easily managed parts called data shards [30]. Often, it can be relatively easy to shard data. This way, a lot of transactions will get validated at the same time on the Ethereum network. In connection to Tutellus, this sharding allows the company to delegate its different tasks so that it maximizes its capacities for its educational functions[54], [31]

Tutellus has become extremely popular in the Spanishspeaking world over 1,000,000 users total. They also provide over 150 education video courses. With many students taking on loans that can range from 20,000-60,000 dollars, there is undoubtedly a marketplace for an application that can reduce those costs.

\section{RQ3: Which Blockchain Features are used by these projects?}

The Goal of this research question is to describe the blockchain features which are used by these projects. The common blockchain features which are used in these projects are listed in Table IV:

- Blockchain-based educational projects are entirely decentralized, rather than the store on a central authority. Educational projects data store in the decentralized blockchain network that will provide transparency and independence in the system. As a blockchain is an open-source of information, it is very important that the collection is being stored in honest and accurate. Projects decentralized management system would additionally facilitate institutions or employers validate quality with merely one click, instead of going through the time consuming and technical method of validating every certification individually.

- Blockchain technology uses the consensus mechanism to validate the new block in a distributed ledger. The recognized projects used a consensus mechanism like POS, DPOS, POW. Hence, this feature of blockchain is also the path of these projects.

- The educational projects focus on the immutable record. Once information is stored in the network, it cannot be changed until the 51 percent of the total node agreed to change.

- Blockchain-based educational projects are open source. The projects are a supportive environment, always open to everyone, and ensure complete transparency.

- Only SGE provides anonymous data. This feature inverse the traceability, Users can store personal data without using any encryption method

- Censorship Resistant means no alteration of data, no rewrite. But everyone can see every transaction. But SGE can censor the transaction due to its anonymity features.

\section{RQ4: What Services are being Offered by the Existing Educational Projects using Blockchain Features?}

The basic purpose of this research question is to identify the services which are being offered by Educational projects using blockchain features. First of all, we thoroughly studied the given research projects. After being analyzed deeply, we figure out the result which is given below in Fig. 3 .

Table V presents a comparison between blockchain-based educational projects. There many advantages of all the technologies, which can be combined, and get an improved outcome. 
TABLE V. Services Provided by the Projects

\begin{tabular}{|c|c|c|c|c|c|c|c|c|c|}
\hline $\begin{array}{l}\text { Projects / } \\
\text { Services }\end{array}$ & Edge-Coin & $S G E$ & $T M P$ & SuccessLife & Tutellus & Blockerts & Grad base & EchoLink & OriginStamp \\
\hline Content Library & Yes & Yes & Yes & Yes & Yes & & & & \\
\hline Store personal data & Yes & Yes & Yes & Yes & & Yes & Yes & Yes & Yes \\
\hline E-Certficate & Yes & Yes & Yes & Yes & & Yes & Yes & & \\
\hline Scoring system & & Yes & Yes & & & & & & \\
\hline B2B apporach & Yes & Yes & & Yes & Yes & & & Yes & \\
\hline Token system & Yes & & & Yes & Yes & Yes & Yes & & \\
\hline Coooperative learning & Yes & & & & Yes & & & & \\
\hline Job opportunites & & Yes & & & Yes & & Yes & & \\
\hline Providing Feedback services & & & & & Yes & & & & \\
\hline
\end{tabular}

The educational projects have a lot of benefits; especially the decentralized approach solves a lot of problems regarding security, paperwork reductions, and job issues. Adopting a standardized blockchain to process several billions of transactions between devices will fundamentally reduce the expenses related to maintaining large centralized data centers and will appropriate calculation and capacity needs over the billions of devices. This will prevent avoid in any single node in the network from conveying the whole system to an ending collapse. These projects are used to connect a wide range of students and teachers for the last few years and will keep on supporting small scale IoT systems. In any case, it won't be capable of providing the needs to broaden the blockchain system in education in the future.

TABLE VI. SERVices OfFEREd by BLOCK-CHAin FeATURES

\begin{tabular}{ll}
\hline Services offered by Projects & \multicolumn{1}{c}{ BC Features } \\
\hline Content Library & Transactiuon Rate, Decentralized \\
\hline Store personal data & Anomity \\
\hline E-Certficate & Transaction Rate \\
\hline Scoring system & Smart Contract \\
\hline B2B apporach & Smart Contract \\
\hline Token system & $\begin{array}{l}\text { Smart Contract, Currency, } \\
\text { Consensus Mechnism }\end{array}$ \\
\hline Coooperative learning & Decentralized, Smart Contract \\
\hline Job opportunites & Smart Contract \\
\hline Providing Feedback & Traceability \\
services & \\
\hline
\end{tabular}

Table VI shows the services that are being offered using blockchain features.

Content Libary: Massive records offered the blockchainbased educational projects by using the blockchain Features transaction rate and decentralization. This service is a platform-independent, which means that if a customer leaves content at one point in one device, he/she continue studying the material at another device.

Store Personal data: Customer can store the personal data into two ways, Encryption method, The transaction that is stored on the network show everyone but in encryption form. Data is encryption by using different codes, and all the projects provide this kind of data security. Second is the anonymous method this technique is utilized only sony global education, unknown transaction store secretly nobody saw the transaction the single customer can see this.

E-Certificate: Existing blockchain provides us with a facility of e certificates by using blockchain features transaction rate. E-certificate is a digital certificate that adopts a digital signature; the client can accomplish it by utilizing the public and private key.

Scoring System: Grades are provided according to the performance of students by using blockchain feature smart contract. Just sony global, and TeachMePlease gives a service of the scoring system. On the off chance that the understudy's performance is adequate, at that point, the evaluation is superb generally ordinary.

B2B approach: It is direct contact starting with one business then onto the next, blockchain helps this System as a result of its decentralized nature. Two business is connected with each other; This service is offered by utilizing the blockchain feature smart contract.

Token System: The token framework could be utilized with the end goal of the transaction and for a reward. Various methods and components are utilized for this, and this service is being offered by feature, currency smart contract and consensus mechanism feature.

Cooperative learning: People learn together in the same place. They talk about the things and create the new thoughts block-chain-based educational projects gave that sort of condition by using blockchain features decentralized and smart contract.

Job opportunities: Block-chain-based educational projects give occupations to individuals. They provide them with the opportunities of work to the certified and skilled person, So, the blockchain reduces the unemployment by using smart contract.

Providing Feedback services: Tutellus Block-chain based educational project provide the facility to the people to give their feedback for that purpose traceability feature is used.

\section{CONClusion}

In this study, we have a trend to map out by using a systematic literature approach that can be useful in this regard. We have perceived suitable blockchain-based educational projects and also presented a comparison between the features of these projects. The implementation of the projects in the field of education has resolved the common issues of the educators. Since this technology is on its initial experimental stages and needs to go through evaluation processes. This technology can bring much more innovation in the future.

\section{REFERENCES}

[1] Nakamoto, S. (2008). Bitcoin: A peer-to-peer electronic cash system. 
[2] Pilkington, M. (2016). 11 Blockchain technology: principles and applications. Research handbook on digital transformations, 225. *

[3] Conoscenti, M., Vetro, A., \& De Martin, J. C. (2016, November). Blockchain for the Internet of Things: A systematic literature review. In 2016 IEEE/ACS 13th International Conference of Computer Systems and Applications (AICCSA) (pp. 1-6). IEEE.

[4] Crosby, M., Pattanayak, P., Verma, S., \& Kalyanaraman, V. (2016). Blockchain technology: Beyond bitcoin. Applied Innovation, 2(6-10), 71

[5] Samaniego, M., \& Deters, R. (2016, December). Blockchain as a Service for IoT. In 2016 IEEE International Conference on Internet of Things (iThings) and IEEE Green Computing and Communications (GreenCom) and IEEE Cyber, Physical and Social Computing (CPSCom) and IEEE Smart Data (SmartData) (pp. 433-436). IEEE

[6] Bhowmik, D., \& Feng, T. (2017, August). The multimedia blockchain: A distributed and tamper-proof media transaction framework. In 2017 22nd International Conference on Digital Signal Processing (DSP) (pp. 1-5). IEEE.

[7] Lemieux, V. L. (2016). Trusting records: is Blockchain technology the answer?. Records Management Journal, 26(2), 110-139

[8] Iansiti, M., \& Lakhani, K. R. (2017). The truth about blockchain. Harvard Business Review, 95(1), 118-127*

[9] Yumna, H., Khan, M. M., Ikram, M., \& Ilyas, S. (2019, April). Use of Blockchain in Education: A Systematic Literature Review. In Asian Conference on Intelligent Information and Database Systems (pp. 191202). Springer, Cham.

[10] M.B. Hoy, "An Introduction to the block-chain and Its Implications for Libraries and Medicine,” Med. Ref. Serv. Q., vol. 36, no. 3, pp. 273-279, 2017. *

[11] Yli-Huumo, J., Ko, D., Choi, S., Park, S., \& Smolander, K. (2016). Where is current research on blockchain technology? a systematic review. PloS one, 11(10), e0163477

[12] Makridakis, S., Polemitis, A., Giaglis, G., \& Louca, S. (2018). Blockchain: The Next Breakthrough in the Rapid Progress of AI In Artificial Intelligence-Emerging Trends and Applications. IntechOpen.

[13] Yang, X., Li, X., Wu, H., \& Zhao, K. (2017). The application model and challenges of blockchain technology in education. Modern distance education research, (2), 34-45

[14] Grather, W., Kolvenbach, S., Ruland, R., Schütte, J., Torres, C., Wendland, F. (2018). Blockchain for education: lifelong learning passport. In Proceedings of 1st ERCIM Blockchain Workshop 2018. European Society for Socially Embedded Technologies (EUSSET) *

[15] Cheng, J. C., Lee, N. Y., Chi, C., \& Chen, Y. H. (2018, April). Blockchain and smart contract for digital certificate. In 2018 IEEE international conference on applied system invention (ICASI) (pp. 10461051). IEEE.

[16] Turkanovic, M., Holbl, M., Kosic, K., Hericko, M., \& Kamisalic, A. (2018). EduCTX: A blockchain-based higher education credit platform. IEEE Access, 6, 5112-5127

[17] Sharples, M., \& Domingue, J. (2016, September). The blockchain and kudos: A distributed system for educational record, reputation and reward. In European Conference on Technology Enhanced Learning (pp. 490-496). Springer, Cham.

[18] Albeanu, G. (2017). Blockchain technology and education. On Virtual Learning, 271.

[19] Rooksby, J., \& Dimitrov, K. (2017). Trustless education? A blockchain system for university grades. In New Value Transactions: Understanding and Designing for Distributed Autonomous Organisations, Workshop at DIS

[20] Vukolic, M. (2015, October). The quest for scalable blockchain fabric: Proof-of-work vs. BFT replication. In International workshop on open problems in network security (pp. 112-125). Springer, Cham.

[21] McConaghy, T., Marques, R., Muller, A., De Jonghe, D., McConaghy, T., McMullen, G., ... \& Granzotto, A. (2016). BigchainDB: a scalable blockchain database. white paper, BigChainDB.

[22] Zheng, Z., Xie, S., Dai, H., Chen, X., \& Wang, H. (2017, June). An overview of blockchain technology: Architecture, consensus, and future trends. In 2017 IEEE International Congress on Big Data (BigData Congress) (pp. 557-564). IEEE.

[23] Biswas, K., \& Muthukkumarasamy, V. (2016, December). Securing smart cities using blockchain technology. In 2016 IEEE 18th international conference on high performance computing and communications; IEEE 14th international conference on smart city; IEEE 2nd international conference on data science and systems (HPCC/SmartCity/DSS) (pp. 1392-1393). IEEE

[24] Alharby, M., \& van Moorsel, A. (2019). BlockSim: A Simulation Framework for Blockchain Systems. ACM SIGMETRICS Performance Evaluation Review, 46(3), 135-138.

[25] Twesige, R. L. (2015). A simple explanation of Bitcoin and Blockchain technology.

[26] X. Liang, S. Shetty, D. Tosh, C. Kamhoua, K. Kwiat, and L. Njilla, "ProvChain: A Blockchain-Based Data Provenance Architecture in Cloud Environment with Enhanced Privacy and Availability," Proc. 2017 17th IEEE/ACM Int. Symp. Clust. Cloud Grid Comput. CCGRID 2017, pp. 468-477, 2017.

[27] D.J. Skiba, "The Potential of Blockchain in Education and Health Care," Nurs. Educ. Perspect., vol. 38, no. 4, pp. 220 221, 2017.

[28] Chen, G., Xu, B., Lu, M., Chen, N. S. (2018). Exploring block-chain technology and its potential applications for education. Smart Learning Environments, 5(1), 1.

[29] Aste, T., Tasca, P., \& Di Matteo, T. (2017). Blockchain technologies: The foreseeable impact on society and industry. Computer, 50(9), 18-28.

[30] Lin, I. C., \& Liao, T. C. (2017). A Survey of Blockchain Security Issues and Challenges. IJ Network Security, 19(5), 653-659.

[31] Team, H. Technical Whitepaper.

[32] A. Grech and A. F. Camilleri, Blockchain in Education. 2017.

[33] Nofer, M., Gomber, P., Hinz, O., \& Schiereck, D. (2017). Blockchain. Business \& Information Systems Engineering, 59(3), 183-187.

[34] E.Buterin, "A next-generation smart contract and decentralized application platform," Etherum, no. January, pp. 1 36, 2014.

[35] Swan, M. (2015). block-chain: Blueprint for a new economy. " O'Reilly Media, Inc

[36] Mohanty, D. (2018). Ethereum Use Cases. In Ethereum for Architects and Developers (pp. 203-243). Apress, Berkeley, CA.

[37] Ocheja, P., Flanagan, B., \& Ogata, H. (2018, March). Connecting decentralized learning records: a blockchain based learning analytics platform. In Proceedings of the 8th international conference on learning analytics and knowledge(pp. 265-269). ACM

[38] Hepp, T., Schoenhals, A., Gondek, C., \& Gipp, B. (2018). OriginStamp: A blockchain-backed system for decentralized trusted timestamping. itInformation Technology, 60(5-6), 273-281.

[39] Soucy, C. (2018). The Changing Tide of Corporate Social Responsibility.

[40] Martos Carrion, E. (2018). Historico-Sociological Analysis of the Sharing Economy from its Early Digital Foundations to the Present.

[41] Martinez-Polo, J., Martinez-Sanchez, J. T., \& Vivo, J. M. N. (2016). Participation and sharing economy: The spanish case of\# compartirmola. In Entrepreneurship, Business and Economics-Vol. 1 (pp. 15-22). Springer, Cham

[42] Kuvshinov, K., Nikiforov, I., Mostovoy, J., Mukhutdinov, D., Andreev, K., \& Podtelkin, V. (2018). Disciplina: Blockchain for Education.

[43] Arenas, R., \& Fernandez, P. (2018, June). CredenceLedger: A Permissioned Blockchain for Verifiable Academic Credentials. In 2018 IEEE International Conference on Engineering, Technology and Innovation (ICE/ITMC) (pp. 1-6). IEEE..

[44] Chan, S. Blockchain Based Professional Networking and Recruiting Platform.

[45] Kitchenham, B., \& Charters, S. (2007). Guidelines for performing systematic literature reviews in software engineering.

[46] S.E. Group, "Guidelines for performing Systematic Literature Reviews in Software Engineering," 2007.

[47] Elendner, H., Trimborn, S., Ong, B., \& Lee, T. M. (2016). The crosssection of crypto-currencies as financial assets: An overview (No. 2016038). SFB 649 Discussion paper.

[48] Kiayias, A., Russell, A., David, B., \& Oliynykov, R. (2017, August). Ouroboros: A provably secure proof-of-stake blockchain protocol. In Annual International Cryptology Conference (pp. 357-388). Springer, Cham. 
[49] King, S., \& Nadal, S. (2012). Ppcoin: Peer-to-peer crypto-currency with proof-of-stake. self-published paper, August, 19. Evaloation process

[50] Gromovs, G., \& Lammi, K. (2017). Blockchain and internet of things require innovative approach to logistics education. Transport Problems, 12.

[51] Vasin, P. (2014). Blackcoin's proof-of-stake protocol v2. URL: https://blackcoin. co/blackcoin-pos-protocol-v2-whitepaper. pdf, 71.

[52] S. King and S. Nadal, "Ppcoin: Peer-to-peer crypto-currency with proofof-stake," Self-Published Paper, August, vol. 19, 2012.

[53] Reyna, A., Martín, C., Chen, J., Soler, E., \& Díaz, M. (2018). On blockchain and its integration with IoT. Challenges and opportunities. Future Generation Computer Systems, 88, 173-190.

[54] Luu, L., Narayanan, V., Zheng, C., Baweja, K., Gilbert, S., \& Saxena, P. (2016, October). A secure sharding protocol for open blockchains. In Proceedings of the 2016 ACM SIGSAC Conference on Computer and Communications Security (pp. 17-30). ACM.

[55] Crespo, A. S. D. P., \& García, L. I. C. (2017). Stampery Blockchain Timestamping Architecture (BTA)-Version 6. arXiv preprint arXiv:1711.04709. 